\title{
F2C - An Innovative Approach to Use Fuzzy Cognitive Maps (FCM) for the Valuation of High-Technology Ventures
}

\author{
Peter Heydebreck ${ }^{1}$, Magnus Klofsten ${ }^{2}$ and Lars Krüger ${ }^{3}$ \\ ${ }^{1}$ Inno AG, Karlsstrasse, Karlsruhe \\ ${ }^{2}$ Department of Management and Industrial Development, PIE / HELIX, \\ University of Linköping, Linköping Sweden \\ ${ }^{3}$ Engage - Key Technology Ventures AG, Rostock / Germany
}

\begin{abstract}
We propose a novel method - F2C - that can process the only information available in the early stages of a young high-technology venture: linguistic expert knowledge. This knowledge is the basis for the determination of an appropriate discount rate for the valuation of high-technology ventures in early stages of venture capital investments. A specific tool named Fuzzy Cognitive Map (FCM) is used therein to capture and depict the expert knowledge. Consequently, the FCM is transformed into a system of Fuzzy Inference Systems. These systems allow eventually obtaining a crisp value for the crucial parameter, the discount rate $r$. First practical applications were carried out valuing a biotechnology venture. The obtained results are promising, though the need for further research efforts became obvious.
\end{abstract}

Keywords: Fuzzy Cognitive Map, Venture Valuation, Fuzzy Systems

\section{Introduction}

There is a substantial need with key decision makers for support instruments that are robust, reliable, and above all, applicable in business real life. Particularly, in the Venture Capital world, decisions that are based on unreliable or even wrong information can lead to significant losses of the invested money. An applicable support tool is particularly essential when it comes to valuing a young high-technology venture before an investment transaction. The inherent peculiarities of small hightechnology ventures in venture capital transactions are analyzed exemplarily in [13].

Due to the lack of historic data, tangible assets, and the limited comparability to grown mature companies even in the same industry field, there is a need for specific valuation methods for young hightechnology companies. A concept that has experienced a broad acceptance with practitioners in the recent years is based on the time-value-of-money-concept. A present value $P V$ of a company is the result of the added discounted cash flow $C F$ the company is going to earn in the next $i$ periods (Equation 1).

$$
P V=\sum_{n=1}^{i} \frac{C F_{i}}{(1+r)^{i}}
$$

Equation 1: Present Value of Future Cash Flows

Copyright (C) 2011 Peter Heydebreck, Magnus Klofsten and Lars Krüger. This is an open access article distributed under the Creative Commons Attribution License unported 3.0, which permits unrestricted use, distribution, and reproduction in any medium, provided that original work is properly cited. Contact author: Lars Krüger e-mail: l.krueger@engage-ventures.com 
Although being rather simple, the challenge lies in the estimation of appropriate parameters. Besides the uncertainty of the future cash flow, the most significant impact on the result has the discount rate $r$. Particularly with valuing high-technology ventures, the discount rate not only reflects the minimum return rate expected by the investor, but also includes compensation for the risk taken by the investor and the added value the investor provides by, for example, management support and access to qualified networks. Even a premium for the limited liquidity of the early stage investment in comparison to conventional investments is oftentimes required (see Figure 1).

\section{Problem}

There is no practicable, distinct and unambiguous method to determine the discount rate $r$ especially in the context of early stage venture valuation of hightechnology companies. In particular, there exists hardly a method that explicitly accounts for the different components of $r$. In Figure 2, it becomes obvious that nondeterministic subjective approaches such as Best Practices or Minimum Expected Returns dominate. Although being fast and easy to apply, these procedures are always prone to subjective misjudgements. In addition, they generally lack transparency and unambiguity. Furthermore, they do not support explicitly in differentiating between the relevant components of the discount rate $r$.

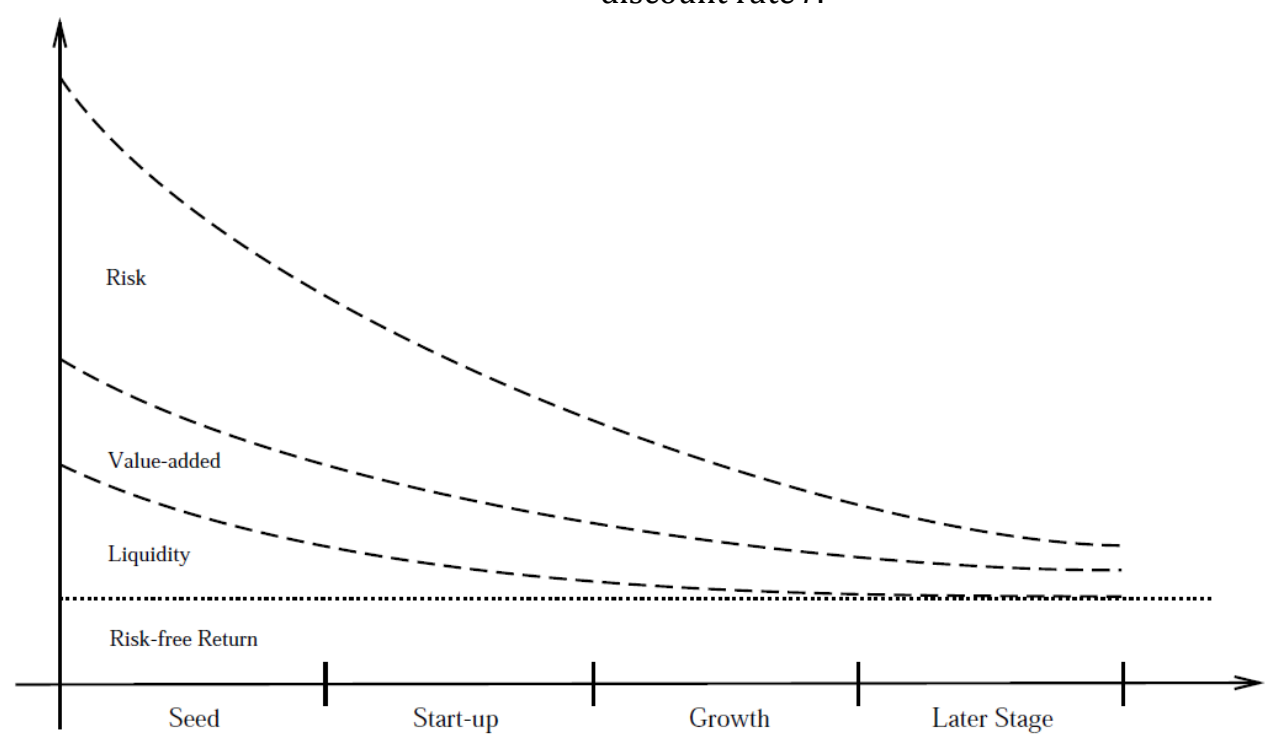

Figure 1: Composition of the Discount Rate $R$ over the Different Stages of Venture Capital

The only theoretically founded objective method based on the Capital Asset Pricing Model (CAPM) is obviously not very common with practitioners as the frequency of use indicates (see Figure 2). This model allows the determination of a theoretically appropriate rate of return of an asset $r$. In detail, the model takes the asset's sensitivity to non-diversifiable risk as well as the expected return of the market $r_{M}$ and the expected return of a theoretically risk-free asset $r_{\mathrm{f}}$ into account. The risk premium is given by the sensitivity to the non-diversifiable market risk $(\beta)$ and the return of the market $\left(r_{\mathrm{M}}\right)$. The appropriate discount rate $r$ is then calculated by $r=r_{\mathrm{f}}+\left(r_{\mathrm{M}} * \beta\right)$. This fundamental feature principally corresponds with the previously mentioned composition of an adequate return-rate. The limited dissemination of this method, however, may be generally due to the harsh assumptions of the underlying theoretical model and the need for appropriate data to obtain meaningful results. 


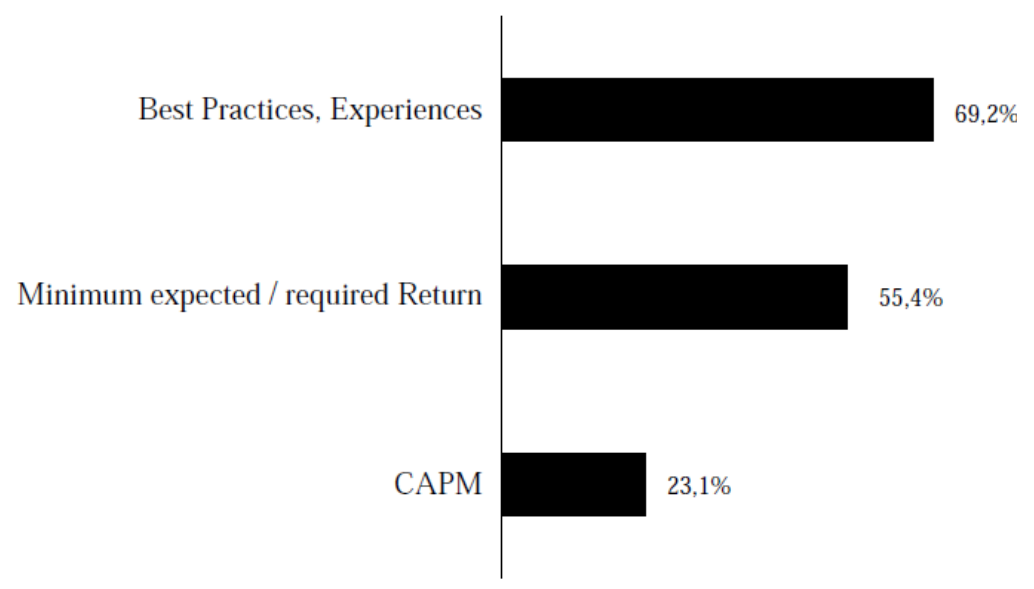

Figure 2: Frequency of Use - Conventional Methods for the Determination of $r$ [11]

\section{State of the Art}

The current research still focuses mainly on the application of the CAPM. The effectiveness and suitability of this model, however, it has been controversially criticized since its appearance (see exemplarily [3], [4], [5], or [6]). But despite the obvious limitations, the CAPM is still used to model and calculate general characteristics of Venture Capital investments (see exemplarily [7], [8]), although, its limited applicability in the field of venture valuation, has been already revealed. Particularly, the clear and comprehensible determination of the parameter _ in early stage venture valuation is seen as extremely challenging: usually $\beta$ s can be easily calculated from the historic returns if the company is publicly traded. High-Technology ventures, however, are not publicly traded. In addition, due to their youthfulness, there is no meaningful historic data on its development. Usually, in such case, there is no historic data available for the $\beta$ of comparable publicly traded companies or even industry-specific betas $(\beta)$ can be used. But these approaches are rather meaningless since the risk profile of known (i.e. mature) companies is not comparable to young ventures [12], [2].

Despite this obvious research gap, there haven been only a few endeavours made to present a tailor-made solution particularly for early stage ventures. One of the most recent approaches is proposed by Sung [1]. The author presents an attempt that directly addresses the issue of a riskadjusted discount rate for the valuation of small-sized technology firms. Particularly, he focuses on the significance of the technological risk in the context of hightechnology venture valuation.

Although aiming at an obvious research gap, this paper does not completely resolve the described shortcomings. In fact, it suffers from the highlighted deficits as well since this work principally bases upon the disputable CAPM and its corresponding enhancement, the Weighted-Average-Costof-Capital approach (WACC). In detail, the paper mainly concentrates on elaborating a statistical framework to estimate more precisely the $\beta$ parameter out of data available for comparable markets and companies. Besides this statistical assistance, the venture's technological risk is captured only by a ranking according to the actual value of three criteria: technology competitiveness, capability of commercialization, and R\&D-support organization. As a consequence, this method cannot help overcoming the challenges associated with high-technology venture valuation. It namely relies on the assumption that by using the CAPM approach, an appropriate discount rate can be obtained. The only thing that matters is 
the quality of guessing the parameters. Moreover, this paper also neglects taking explicitly the different components of the discount rate into account.

\section{Aim of this Paper}

We propose a novel approach that might help overcoming the aforementioned shortcomings with conventional discount rate determination in the context of young high-technology venture valuation. By applying fuzzy methods we try to avoid making unrealistic model assumptions, and thus, obtaining unrealistic results. Due to this well-founded theory, our method offers traceability, clearness, and robustness. Furthermore, the use of expert knowledge as the only reasonable source of information available shall provide broad applicability. The actual influence of the different premiums on $r$ is explicitly accounted for by integrating multi-causal and non-linear coherences between. The actual values of the factors and their interconnectedness are derived from the only information reasonably available: the assessment of the key personal involved in the actual venture: entrepreneurs, scientists, investors, and neutral mediators.

To capture the complex, multi causal relationships, a known concept is used: Fuzzy Cognitive Maps (FCMs). But, in contrast to conventional applications (Knowledge Mapping, Decision Support), the FCM concept is enhanced by an individually developed method to calculate a "crisp" value out of the captured knowledge. It is based upon rule-based Fuzzy Inference Systems (FIS) and a specific methodology to transform an FCM into a network of standardized FIS.

\section{Contribution}

Our contribution to the current state-ofthe-art contacts two dimensions. First of all, we use a novel method to transform methodically clear and unambiguous an FCM into a network of FIS. This can make our approach to an enhancement to and concretion of the procedures proposed by Eloff et al. [22] and Khan and Khor [14]. Our framework, though, is an alternative proposal to Carvalho and Tomé [17]. Second, by using this methodological framework, we propose a completely different way to determine an appropriate discount rate $r$ for venture valuation methods. Our approach can be seen as an entirely novel alternative to conventional methods. This may open up completely new paths to venture valuation particularly at early stage venture capital investments.

\section{Structure}

In Section II, the fundamentals of FCMs are explained. Upon these preliminaries, the specific methodology is presented that allows a transformation of an FCM into a FIS. In section IV, we report on a first practical application of this method. We describe our procedure and the results attained so far. In the final section $V$, we review our results so far. In addition we briefly outline the work that is still to be done in order to present a fully equivalent alternative to conventional methods.

\section{Methodology}

\section{Preliminaries}

FCMs are fuzzy graph structures for representing conceptual pictures of interconnected complex systems. They work by capturing and representing causeand-effect-relationships. A FCM pictures the way of human thinking and incorporates intuitive and heuristic expert knowledge. They are a combination of Neuronal Networks and Fuzzy Logic. FCMs consist of various nodes (concepts) connected by directed graphs (edges) with feedback. These connections stand for the causal relationship between the nodes. Causal relations always involve change: the result of a causal effect is always a variation in one or more concepts/ nodes. 


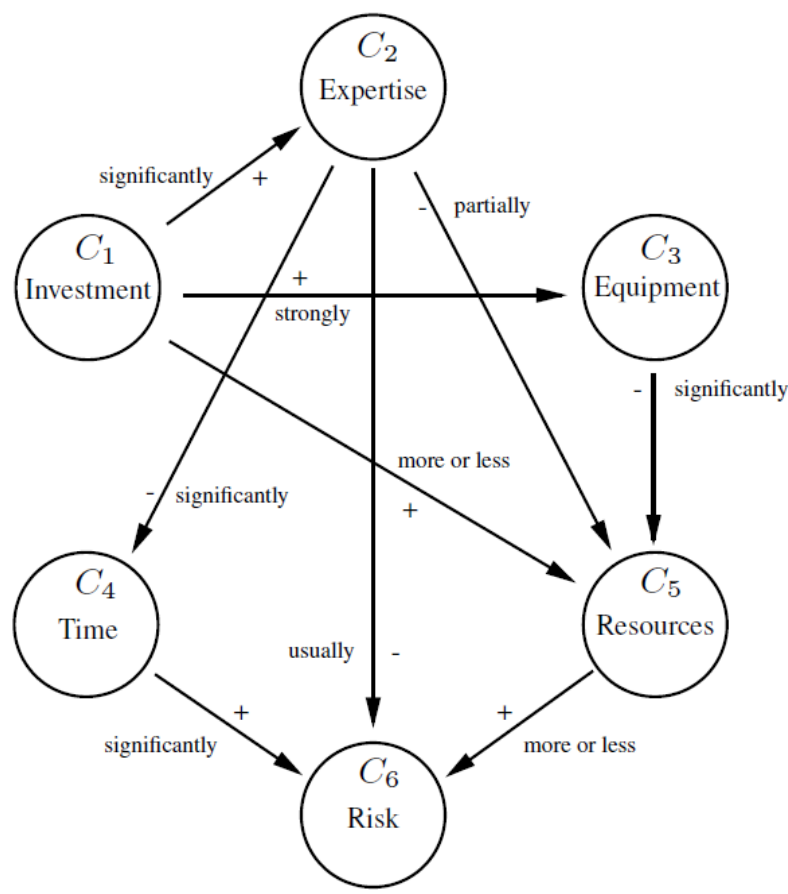

Figure 3: Exemplary Fizzy Cognitive Map

Therefore, FCMs show the variation of a concept's value, not the concept's absolute value. Each node has a fuzzy value ranging from $[-1 ; 1]$. Each edge is associated to a fuzzy weight $\mathrm{w} \square[-1 ; 1]$. A positive weight stands for a causal increase; a negative weight indicates an opposite causal decrease (See Figure 3 for an exemplary FCM). The causal structure of an FCM is formally expressed by the corresponding Adjacency Matrix E:

$$
E=e_{i j}^{(2)}=\sum_{k=1}^{n} w_{i k} \times w_{k j}
$$

\section{Equation 2: Adjacency Matrix E}

The capability to capture the characteristics and the behaviour of complex systems has led to two general application forms: Knowledge Mapping (KM) and Decision Support Systems (DSS) [15]. Both application types have been applied in various fields: Politics, Environment issues, IT Security, Diagnostics, and even in Finance [28]. These applications, however, suffer from a major restriction. Almost all current FCM applications are limited to bivalent or trivalent concept values $(0,1)$ or $(-1,0,1)$. These restrictions limit FCMs to the representation of systems that use only simple monotonic and symmetric causal relations between concepts. But many real world causal relations are neither symmetric nor monotonic.
A fuzzy-rule-based approach provides a far more adequate way to model real-world causal relationships. A Fuzzy Inference System (FIS) makes use of fuzzy rules in which real world coherences are tied together, e. g., "IF the technology is relatively new, THEN the risk of the Venture is moderately high". All the rules define patches that cover the characteristic curve of an arbitrary complex system. The better the rules cover the curve, the smarter the artificial intelligence system. In practice, these rules are called rule-ofthumb or heuristics. They reflect an expert's action to control or an observation of a system. But, identifying and formulating rules that fully describe the behavior of complex systems is the key issue in Fuzzy Engineering. But at the same 
time, it is known to be the natural bottleneck of Fuzzy Engineering [25].

Until now, there have been only some approaches to use the knowledge captured in an FCM to design and build a more adequate rule-based Fuzzy system. According to Eloff et al. a FCM is an ideal starting point to derive a rule base form: the edge between two nodes represents a fuzzy IF-Then-Rule [22], [16]. This "practical" approach, however, leaves the user in the lurch when it comes to typical challenges such as multiple causal relations and non-linear causal behaviour. A more sophisticated approach is proposed by Carvalho and Tomé: Rule-Based Fuzzy Cognitive Maps (RB-FCM) [17], [19], [18], [21]. Such a FCM consists of fuzzy nodes (concepts) and a fuzzy rule base which relate nodes. Each concept contains several membership functions which represent the concepts' possible values or the possible change of its values. To adequately model real-world causality (nonlinearity, nonsymmetric opposition, similarity, implication), both authors introduce a novel concept: Fuzzy Causal Relation (FCR) and Fuzzy Carry Accumulation (FCA). These two concepts mainly offer a resort to the problem of causal overflow: what happens to an effect node $\mathrm{C}$, if the two cause nodes A and B effect C "very much" and there is no such a change in the concept value as "more than very much".

In comparison to a classic FCM, the major advantages of the RB-FCM are more flexible modelling of causal relations and improved stability regarding the application for scenario simulation purposes. Though, this approach has also some deficits. It is rather complex and its computation is timeconsuming. Moreover, it does not explicitly address the issue of how to cope with complex multiple causal relations. A slightly different model is proposed by Khan and Khor [15], [14]. In contrast to the RB-FCM, in which the states of the nodes are seen as additive and cumulative so that the state values can be 'carried over' when they exceed a maximum, both authors suggest that each concept has a maximum and a minimum limit. This limit is expressed in form of a weight vector such that the total of the causality is within the interval $[0 ; 1]$. By proposing the Aggregation Operator,

$\mathrm{A}:(\mathrm{c} 1 ; \ldots, \mathrm{ci})=\sum_{i=1}^{n} w_{i} d_{i}$,

they explicitly address the issue of causality in the multiple input case. But, at the same time, they neglect to address the issue how to derive a rule base from an existing expert FCM.

\section{Novel Framework}

1. Interpretation of Nodes and Edges: We try to overcome the previously highlighted shortages of conventional FCM transformation by a specifically developed method [20]. The major assumption of the novel framework is that there is no central rule base that represents the causal relations between the nodes. In contrast, it is assumed that each causal link can be represented by a standardized rule base. A causal link of the causal strength $w_{\mathrm{ij}}$ between two nodes $\mathrm{C}_{\mathrm{i}}$ and $\mathrm{C}_{\mathrm{j}}$ depicted by an arrow in an FCM is represented by a rulebased Fuzzy Inference System FIS $S_{\text {ij }}$ (see Figure 4). Hence, each causal link in an FCM can be translated into a corresponding single-input-single-output Fuzzy Inference System. By applying this procedure, every highly-individual FCM can be translated into a network of Fuzzy Inference Systems.

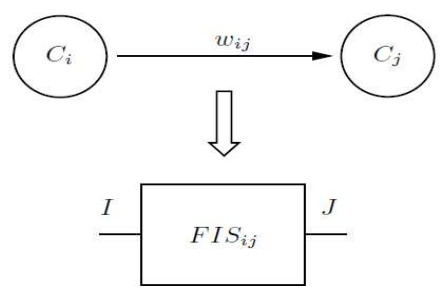

Figure 4: Representation of a Single Causal Link by an FIS 
2. Multiple Causal Inputs: To cope with multiple causal inputs, a method for combining multiple causal inputs is proposed being similar to the aggregation operator A proposed by Khan and Khor
[15], [14]. Initially, all incoming edges are transformed to individual FIS according to the causal weight associated to them. Afterwards, the results of these FIS are combined using the aggregation operation.

$$
\mathrm{A}=\frac{1}{n} \sum_{i=1}^{n} w_{i} d_{i}
$$

\section{Equation 3: Aggegation Operator $A$}

Thereby, the incoming signals can be weighted according to their (assumed) importance (Figure 5). Usually, the signals are all treated equally. The outgoing signal represents the combined causal effect of the preceding cause nodes on the following effect node.

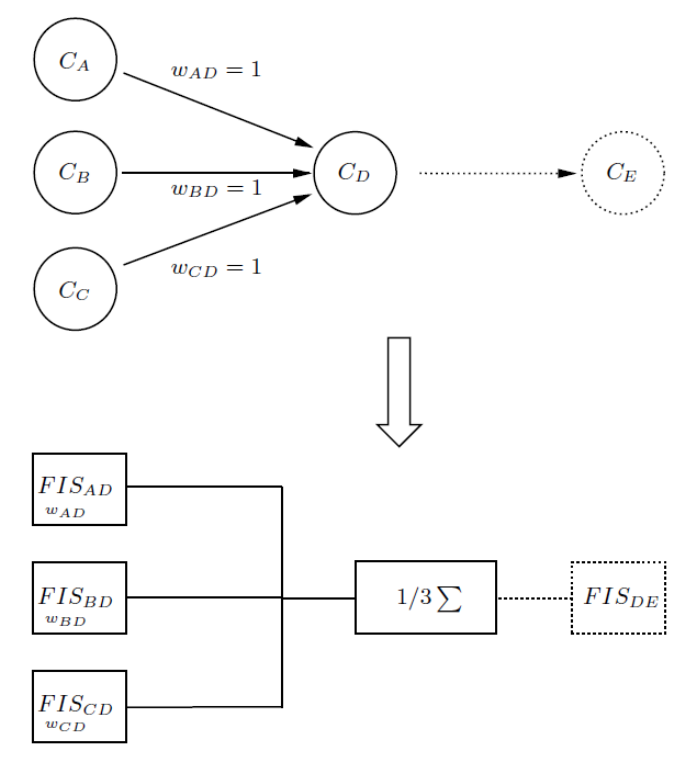

Figure 5: Interpretation of Multiple Causal Links

3. Rule-based Causality: Each of these causalities is represented by a specific rulebased Fuzzy Inference System. The type of causality (positive or negative) as well as its strength is expressed by a specific rule base. The rule base is made for a singleinput-single-output-Fuzzy Inference System. On the one hand, this decreases the calculation power needed to calculate a whole network of FIS. On the other hand, it reduces the risk of rule explosion.

The input and output parameters of the FIS correspond with the Level of Activation of the actual node. They are represented by $I$, whereas $J$ denotes the output parameter level of activation of the effect node. Both parameters are defined on the basic set $X_{I, J}$ $=[-1 ; 1] \square \mathrm{R}$. An input signal $I=1: 0$ stands for the highest activation of the actual concept. It can correspond with the highest growth of the underlying concept. In contrast to that, an input value $I=-1: 0$ expresses the highest activation of the node in the negative sense; it corresponds with the largest decline of the actual concept. The parameters $I$ and $J$ can take 21 parameter values each. Every step at 0.1 is represented by a parameter value. The output signal $J$ equals the value of the concept node $\mathrm{Cj}$ and is, thus, possibly the input signal $I$ of a successive FIS. 
Each parameter value is mapped onto a Fuzzy Set. The corresponding membership function is bell-shaped or Gaussian. This form of membership function provides a more realistic modeling of the underlying concept values and their corresponding membership degrees. At the outer borders of a fuzzy set, bell-shaped membership functions allow for a less harsh end and beginning of the membership. But, the closer the value gets to center $c$ of the fuzzy number, the stronger the membership degree grows. This non-linearity corresponds with the real-world understanding of fuzzy observations. The set of membership functions representing the input and output parameter $I$ and $J$ is shown in Figure 6.

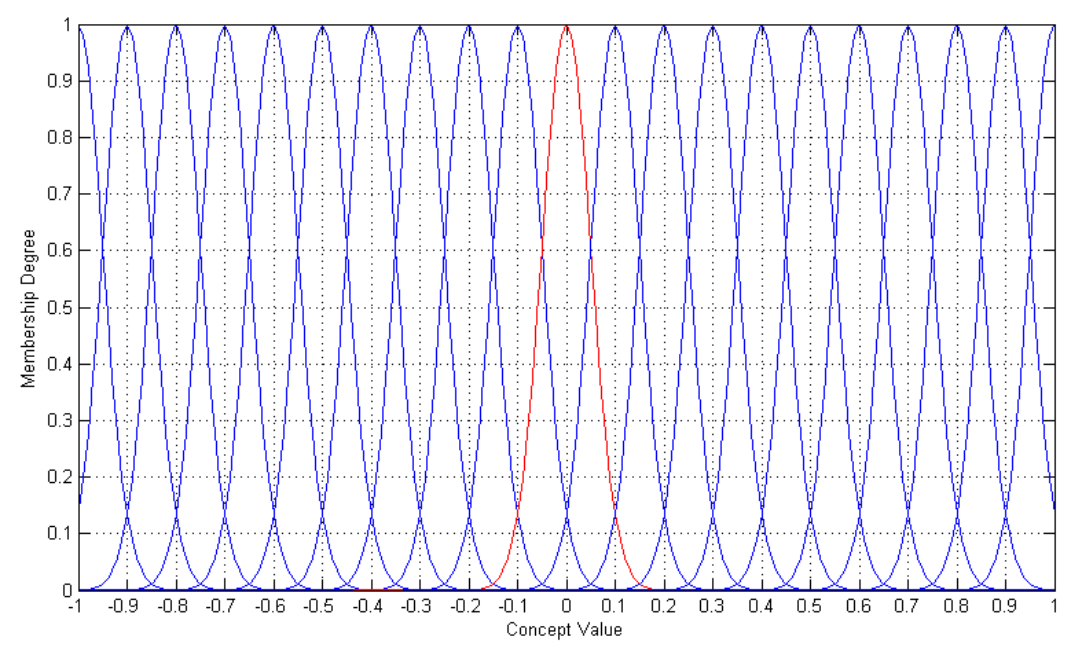

Figure 6: Membership Functions of the Input I and Output J Parameters

Next, the causal linkage between the two parameters has to be defined. To model (non-linear and non-symmetric) causality a specified rule base is designed. Each causality, and hence each causal strength is represented by a specific rule base. The IFpart of the rule consists of only one input $I$. This signal equals the fuzzy number of the concept node value $C_{\mathrm{i}}$. Due to the discretization in steps of 0.1 , the rule base is limited to a reasonable number of rules. Correspondingly, the THEN-part of the rule also consists of only one defuzzified output signal $J$. To determine the individual rule bases, the general understanding of the causal relationship between input and output parameter has to be highlighted. A positive causal relationship between $C_{\mathrm{i}}$ and $C_{\mathrm{j}}$ means that if $C_{\mathrm{i}}$ increases (less/ much) then $C_{\mathrm{j}}$ also increases (less/ much). A positive causal relation that has the causal strength $w_{\mathrm{ji}}<1$ leads, thus, to a weakened increase of $C_{\mathrm{j}}$ in case $C_{\mathrm{i}}$ increases. This weakening effect grows the lower the causal strength is. For example, a concept value of $C_{\mathrm{i}}=1: 0$ may lead only to a concept value $C_{\mathrm{j}}=0: 2$. The same holds true for a decline when there is a positive causal relation. An exemplary rule base representing a positive causal strength $w=$ 1:0 and the corresponding output profile is shown in Figure 7 and Figure 8, respectively. Analogue to this example there are specific rule bases for all possible causal weights $\mathrm{w}$; $\mathrm{w} \square[-1 ; 1]$. In the herein proposed methodological framework, there is a total of 11 specific rule bases (increment of causality is 0.2 , ranging from -1 to 1$)$. 


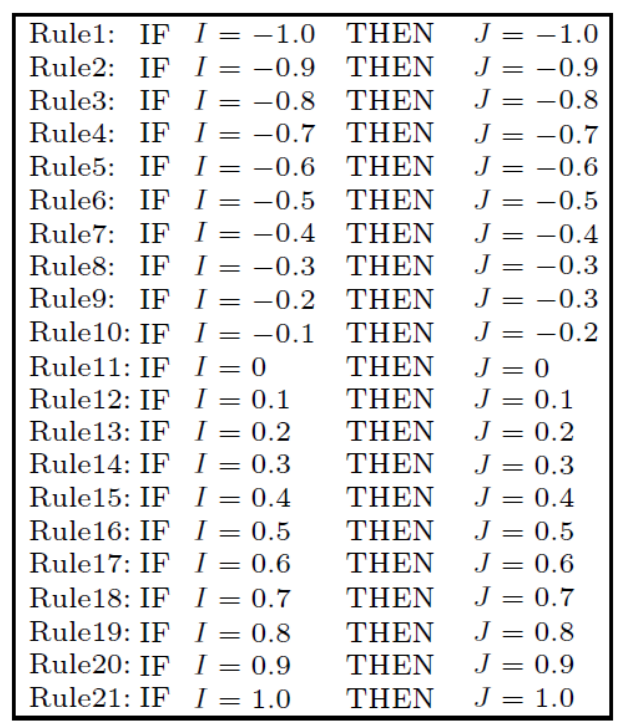

Figure 7: Rule base for causality wij=1.0

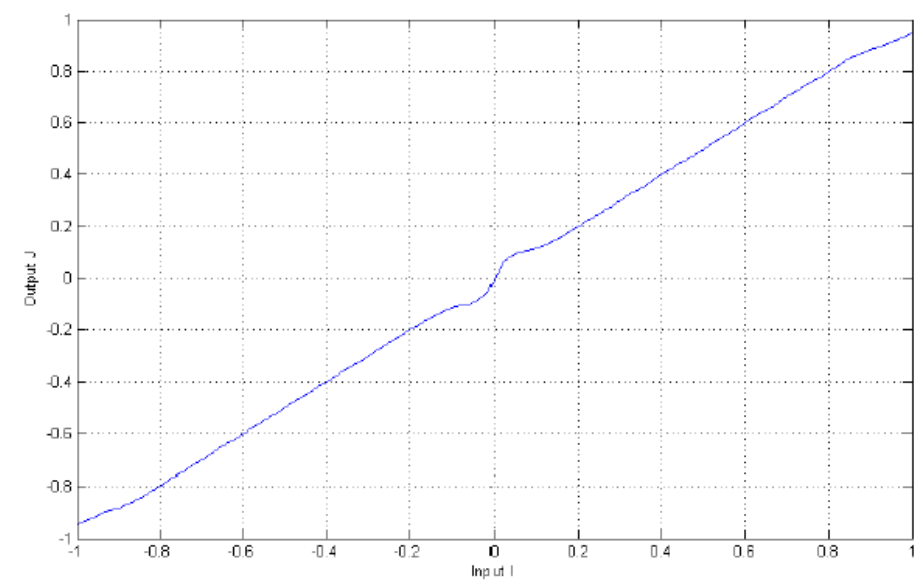

Figure 8: Output Profile of the FIS for Causality wij=1.0

4 Crisp Output: To eventually obtain a crisp output, a specified component is needed: a

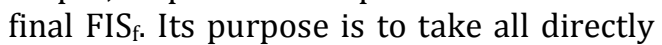
preceding causal links and to 'calculate' a final output value. The input value is obtained by aggregating all incoming causal links (inputs) by using the aggregation operator (Figure 9). Its value ranges from +1 (highest increase) to -1 (highest decrease). The input value is mapped onto the corresponding output value by a specific rule base depending on the actual context. By applying this methodological framework, it is possible to describe and model any complex system and its behavior by means of FCMs. Subsequently, this model of a complex system can be analyzed quantitatively, that is, a crisp value is returned. 


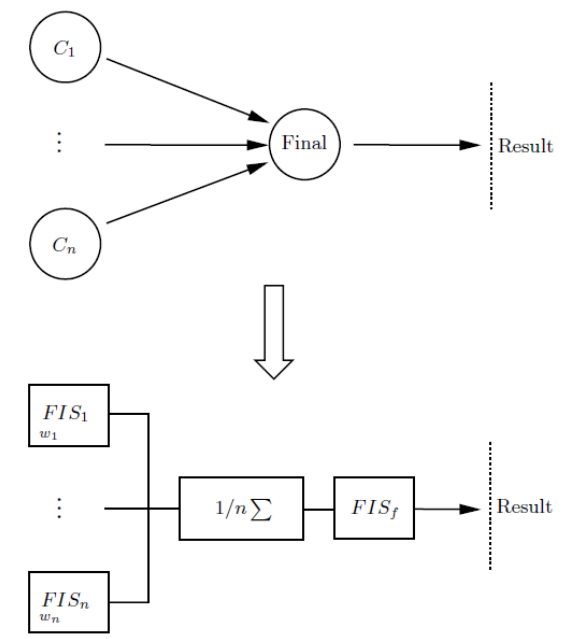

Figure 9: Aggregation and Final FIS - Output of a Crisp Value

\section{Application}

We applied the aforementioned framework to obtain an appropriate discount rate for the valuation of a specific biotechnology venture. Therefore, we substantiated the risk component. We assume that the risk in the early stage of a high-technology venture is inseparably connected with the proof of technology. The company will certainly fail if the technology that shall form the company's basis does not work properly. In this special case, the proof of technology is attained when it is feasible to produce stable and selectively functioning enzymes. For that reason, the simple node Risk is substituted by a more detailed network of different factors that impact the risk of this actual venture. Regarding the detailed Technology Risk, specific starting points are given. The pertinent literature (for example [9], [10]) names three main success factors for the successful development of a young technology venture: Team Experience, Technical Equipment, and Affiliation to a Research Institute (see Figure 10). So, the appropriate discount rate $r$ is influenced not only by factors: Liquidity and Added Value, but also by the detailed Technological Risk. We asked then the involved scientists, investors, and a neutral mediator to identify the risk-influencing aspects and the type and strength of causal relationships between them. Starting from the given nodes, the experts are asked to develop their individual FCMs.

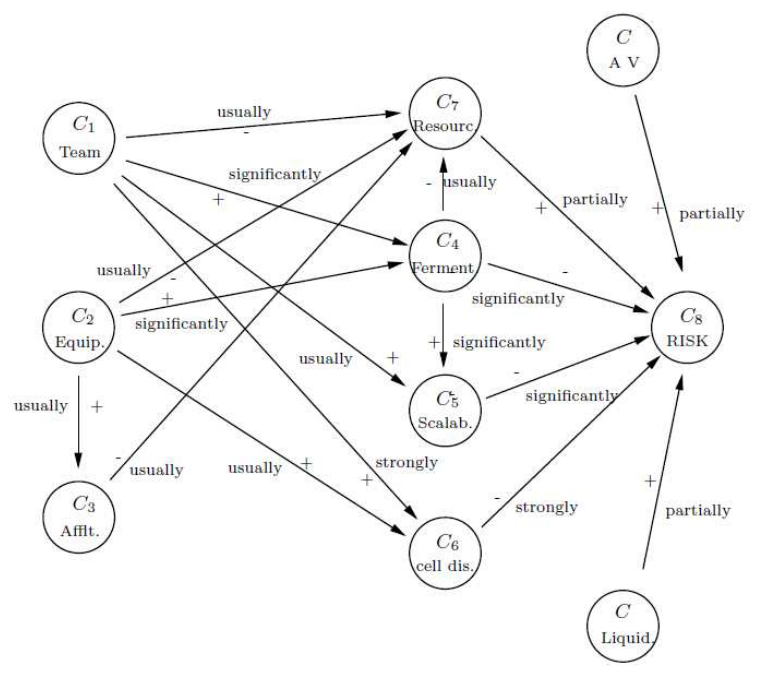

Figure 10: Exemplary FCM - Biotechnology Venture 
The intermediary nodes and the causal connections they create are supposed to eventually link to the final node representing the discount rate $r$. Each of the interviewed experts eventually drew an individual FCM from which the corresponding adjacency Matrices $E_{\mathrm{i}}$ could be easily derived. The final FCM to be transformed is then obtained stepwise. First, a preliminary adjacency matrix is obtained by

$$
E_{\text {pre }}=\frac{1}{i} \sum_{n=1}^{i} E_{i}
$$

\section{Equation 4: Preliminary Adjacency Matrix}

Though, the resulting causal weight $w_{\text {fin }}$ is not a simple product of adding the individual causal weights $w_{\mathrm{ij}}$ of the $\mathrm{i}$ experts. In fact, the causal weights are obtained by a discrete discrimination in a second step. This allows for a consistent conversion of the FCM into a FIS (see Equation 5):

$$
w_{\text {fin }}= \begin{cases}0, & \text { if } 0<w_{i j} \leq 0.1 \\ 0.2, & \text { if } 0.1<w_{i j} \leq 0.3 \\ 0.4, & \text { if } 0.3<w_{i j} \leq 0.5 \\ 0.6, & \text { if } 0.5<w_{i j} \leq 0.7 \\ 0.8, & \text { if } 0.7<w_{i j} \leq 0.9 \\ 1.0, & \text { if } w_{i j}>0.9\end{cases}
$$

\section{Equation 5: Discrete Discrimination of the Causal Weight $W_{\text {fin }}$}

According to the previously highlighted methodological framework, the resulting FCM (see Figure 10) is then transformed into a network of FIS. The 'final' FIS (see Figure 9) is of particular importance. The 'incoming' factors are aggregated according to Equation 3 and subsequently, mapped onto empirically observed discount rates. The mapping is realized by a specific set of rules. The corresponding membership functions for the discount rate $r$ are depicted in Figure 11. This rule base, and thus, the behavior of the final FIS are designed to match the observed correlation between the influencing factors and the required return of Venture Capital investment [11]. A discount rate $r$ can be computed when realistic values ranging from $\square 1$ to 1 are inserted into the starting nodes and the two nodes denoting the remaining components Liquidity and the Value-added premium. These values are also derived by the expert interviews. Their qualitative assessments on the actual value of the starting nodes are translated into numeric values according to Table I. These values trigger the network of FIS. The output is the wanted individual discount rate $r$.

Table 1: Lingusitic and Quantitative Weights (Absolute Values)

\begin{tabular}{|c|c|}
\hline Linguistic Weight & Quantitative Weight \\
\hline \hline More or less & 0.2 \\
Partially & 0.4 \\
Usually & 0.6 \\
Significantly & 0.8 \\
Strongly & 1.0 \\
\hline
\end{tabular}




\section{First Results}

In a first computer-based simulation using the numerical simulation software SIMULINK $^{\text {TM }}$ including the Fuzzy Logic Toolbox we attained a convincing output profile of the final FIS (see Figure 12). This FIS network showed stable behaviour and a crisp final result could be obtained. As it can be seen there is a fundamentally positive coherence between the assumed influence factors and an appropriate discount rate. But, the growth of the discount rate is not constant. In fact, it can be seen that especially, the higher the risk grows; the sharper becomes the increase of the discount rate $r$. This behaviour corresponds with the empirically observed required discount or return rates of Venture Capital investors [11].

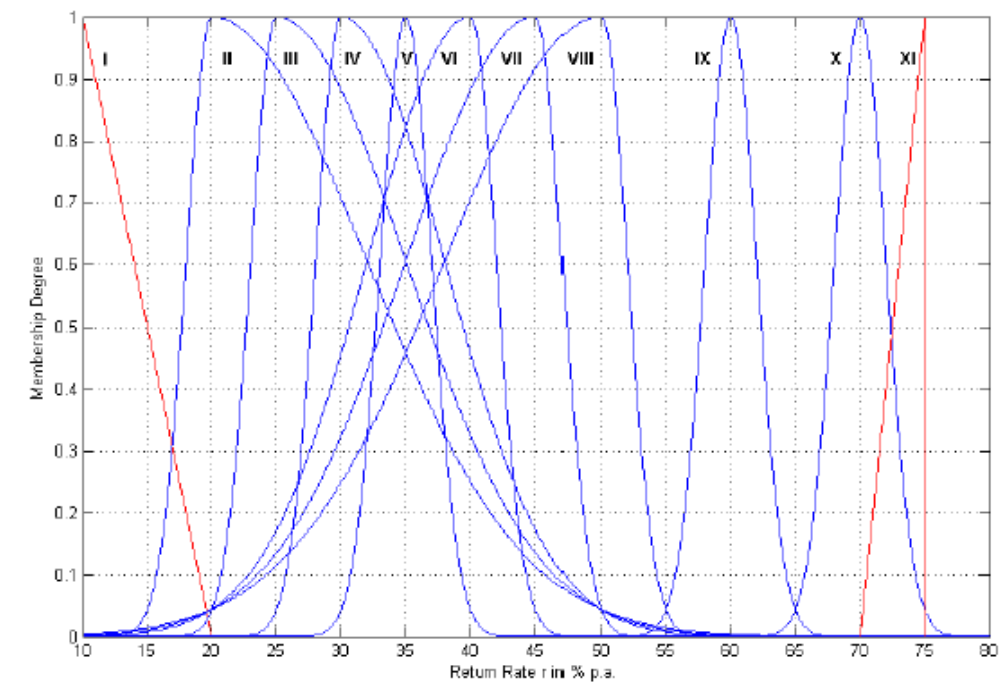

Figure 11: Membership Functions of the Output Parameter Discount Rate $r$

\section{Conclusions}

Our method goes far beyond the conventional qualitative answer to 'what-if' scenarios. In detail, we showed that it is feasible to obtain a crisp value out of linguistic expert information. Based on the previously highlighted methodological framework, it was shown that an FCM is more than a 'hint' from which a rule base for a FIS can be derived. In fact, by using the methodological framework, we achieved that an FCM can be transformed methodologically clear and unambiguously into a network of FIS. A first application was found in the determination of an appropriate discount rate $r$ for venture valuation in the field of early stage hightechnology ventures. By doing so, we could propose an alternative approach to conventional methods for determining an adequate discount rate $r$. The main advantage is that our method does not depend on vast statistical data. In fact, our method only relies on linguistic expert information and waives unrealistic model assumptions. This feature makes our approach unique regarding applicability. The first simulation results confirms the suitability of our approach to model realistically complex systems: the resulting discount rate $r$ shows a behaviour that fundamentally equals the empirically observed correlation between the three components, dominated by Risk, and the discount rate $r$.

Nevertheless, a lot of work is still to be done. Although showing promising results in simulations, the practical superiority has still to be validated in an empirical study. Those studies can also help figuring out when our method can be used complementary or even as a stand-alone solution. 


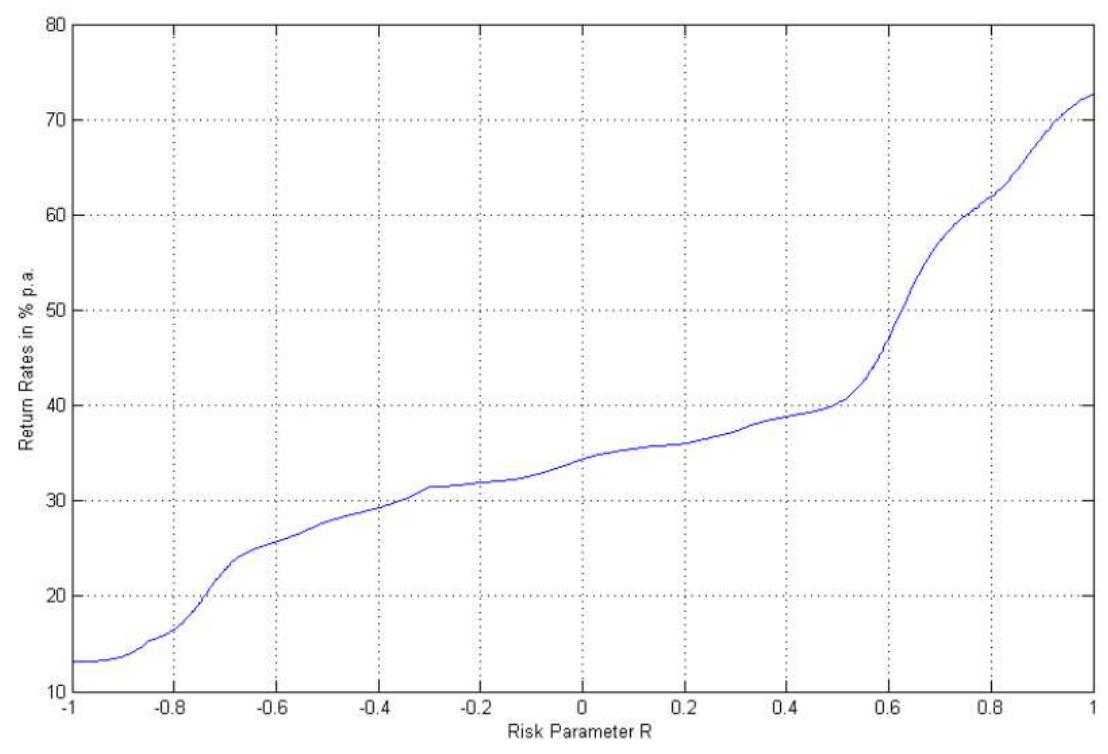

Figure 12: Simulation Results - Discount Rate $r$

\section{Acknowledgements}

The author wishes to thank engage - Key Technology Ventures $A G$ for the intellectual and material support.

\section{References}

Achleitner, A.-K. \& Nathusius, E. (2004). "Venture Valuation and Venture Capital Financing," (German)., Wirtschaftswissenschaftliches Studium, 3, 134-139

Achleitner, A.-K. \& Nathusius, E. (2004a). "Venture Valuation - Valuation of HighGrowth Companies," (German)., SchäfferPoeschel, Stuttgart, Germany

Balder, D. (2004). "Fuzzy Cognitive Maps and their uses as Knowledge Mapping system and Decision Support Systems - A Review of the current state of the practice in the light of the BAMBAS Project," Working Paper, University of Amsterdam, NL, (2004).

Carvalho, J. P. (2002). "Mapas Cognitivos Baseados em Regras Difusas: Modelacao e Simulacao da Dinamica de Sistemas Qualitativos," Instituto Superior Tecnico, Universidade Tecnica de Lisboa, Lisbon,
Portugal.

Carvalho, J. P. \& Tomé, J. A. (2000). "Rulebased Fuzzy Cognitive Maps - Qualitative Systems Dynamics," Proceedings of the 19th International Conference of the North American Fuzzy Information Processing Society (NAFIPS2000)., ISBN 0-7803-62748 ,13-15th July 2000, Atlanta, Georgia, USA, 407-411

Carvalho, J. P. \& Tomé, J. A. (2001). "Expressing Time in Qualitative System Dynamics," Proceedings of the 2001 FUZZIEEE, ISBN 0-7803-7294-X, 2nd-5th December 2001, Melbourne, Australia, 280283

Carvalho, J. P. \& Tomé, J. A. B. (1999). "Rulebased Fuzzy Cognitive Maps and Fuzzy Cognitive Maps - A Comparative Study," Proceedings of the 18th International Conference of the North American Fuzzy Information Processing Society, (NAFIPS99)., ISBN 0-7803-5211-4, 10th12th June 1999, New York, NY USA, 115119

Cochrane, J. H. (2005). "The Risk and Return of Venture Capital," Journal of Financial Economics, 75, (1)., 3-52 
Eloff, J. H. P. \& Smith, E. (2000). "Using Cognitive Modelling for enhanced Risk Assessment in a Health Care Institution," IEEE Intelligent Systems and their Applications, 15, (2)., , 69-75

Eloff, J. H. P. \& Smith, E. (2001). Transaction-based Risk Analysis Using Cognitive Fuzzy Techniques, Proceedings of the 8th Annual Working Conference on Information Security Management \& Small Systems Security (IFI TC-11)., ISBN 0-7923-8626-4, 27-28th September 2001, Las Vegas, Nevada, USA, $141-156$

Fama, E. \& French, K. R. (1996). "The CAPM is Wanted, Dead or Alive," The Journal of Finance, 51, 1947-1958

Fama, E. \& French, K. R. (2002). "The Equity Premium," The Journal of Finance, 57, 637-659

Friend, I. \& Blume, M. E. (1970). "Measurement of Portfolio Performance under Uncertainty," The American Economic Review, 60, 561-575

Gibbons, M. R. (1982). "Multivariate Tests of Financial Models," Journal of Financial Economics, 10, 3-27

Hall, R. E. \& Woodward, S. E. (2007). "The Quantitative Economics of Venture Capital," Working Paper Stanford University,[Retrieved December 22, 2010], http://www.stanford.edu/

rehall/QEVC012707.pdf

Khan, M. S., Chong, A. \& Quaddus, M. (2004). "Fuzzy Cognitive Maps and Intelligent Decision Support - A Review," Journal of Systems Research and Information Science, 26, (2004)., 257-268

Khan, M. S. \& Khor, S. W. (2004). "A Framework for Fuzzy Rule-Based Cognitive Maps," Lecture Notes in Computer Science Vol. 3157/2004, Springer, Berlin Germany, 454-463

Klofsten, M. \& Jones-Evans, D. (1996). "Stimulation of Technology-based small
Firms - A Case of Industry / Industry Cooperations," Technovation, 16, 187-193

Klofsten, M., Jonsson, M. \& Simon, J. (1999). "Supporting the Pre- Commercialisation Stages of Technology-based Firms: The Effects of Small-Scale Venture Capital," International Journal of Venture Capital, 1, (1)., (1999)., 43-52

Kosko, B. (1986). "Fuzzy Cognitive Maps," International Journal of Man- Machine Studies, 24 (1986)., 65-75

Kosko, B. (1994). "Fuzzy Systems as Universal Approximators," IEEE Transactions on Computers, 15, (1994)., 1329-1333

Kosko, B. (1997). "Fuzzy Engineering," Prentice-Hall, Saddle River, New Jersey 